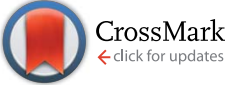

Cite this: RSC Adv., 2017, 7, 6583

Received 29th November 2016 Accepted 6th January 2017

DOI: 10.1039/c6ra27502h

www.rsc.org/advances

\section{A smart NIR fluorescent probe for the highly selective detection of palladium $\dagger$}

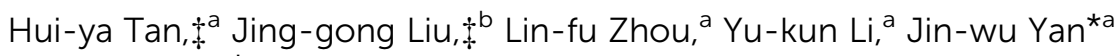 \\ and Lei Zhang*a
}

A tailor-made colorimetric and NIR fluorescent probe for $\mathrm{Pd}^{0}$ was developed by introducing allyl chloroformate into the methylene blue (MB) fluorophore. The probe exhibited vivid color change and significant fluorescence enhancement towards $\mathrm{Pd}^{0}$, accompanied with excellent selectivity and sensitivity. No absorption and fluorescence response could be observed towards other metal ions. The fluorescence intensity of this probe showed a linear response to $\mathrm{Pd}^{0}$ in the concentration range of $0-2$ $\mu \mathrm{M}$ with a detection limit of $5.7 \mathrm{nM}$. Moreover, living cell imaging results indicated that this probe holds promising application prospects for detecting intracellular $\mathrm{Pd}^{0}$ species based on its low cytotoxicity and specific turn-on NIR emission.

\section{Introduction}

Along with the rapid development of modern industry, heavy and transition metals have been widely applied in many fields such as chemistry, biology and environmental science. Palladium, as an important transition metal, plays an important role in various fields such as glass, fine chemical, electronics, and petroleum industries, as well as in automobiles. ${ }^{1-3}$ However, the high level of residual palladium due to the wide exploitation of palladium species has raised palladium contamination, which could cause severe adverse health effects. ${ }^{4,5}$ Therefore, it is urgent to develop highly selective and sensitive methods for palladium detection in environmental and biological samples. Atomic absorption spectroscopy (AAS), inductively coupled plasma mass spectroscopy (ICP-MS), and plasma emission spectroscopy are the conventional approaches for palladium detection, which can achieve accurate and sensitive detection, but expensive facilities, well-controlled experimental conditions and complicated sample-pretreatment procedures are required. ${ }^{6-8}$ In recent years, optical probes, including colorimetric or fluorescent probes, emerged as powerful alternatives to monitor and sense palladium species because of their convenience, on-site detection, ease of manipulation and

\footnotetext{
${ }^{a}$ School of Bioscience and Bioengineering, South China University of Technology, Guangzhou, P. R. China. E-mail: yjw@scut.edu.cn; lzhangce@scut.edu.cn; Tel: +86 2039380678

${ }^{b}$ The Second Affiliated Hospital of Guangzhou University of Chinese Medicine, Guangdong Provincial Academy of Chinese Medical Sciences, Guangzhou, 510006, P. R. China

$\dagger$ Electronic supplementary information (ESI) available: Synthesis and characterization of MB-APC, experimental procedures, and supplemental spectra and graphs. See DOI: 10.1039/c6ra27502h

\$ These authors contributed equally to this work.
}

biological applications. ${ }^{9-13}$ In particular, fluorescent probes with near-infrared (NIR) emission are extremely favourable for bioimaging because of deep penetration and minimum autofluorescence. ${ }^{14,15}$ However, most of the reported probes for palladium display absorbance and emissions in the visible region, which greatly restricts their applications in biological imaging. To this end, it is highly desired and urgent to develop NIR fluorescent probes for selective and sensitive detection of palladium.

Methylene blue (MB), an oxidised phenothiazin compound already FDA-approved for several indications, was widely used as a medication and stain in both clinical and basic research field. ${ }^{\mathbf{1 6 - 1 8}}$ Recently, MB was also applied as NIR imaging agent in

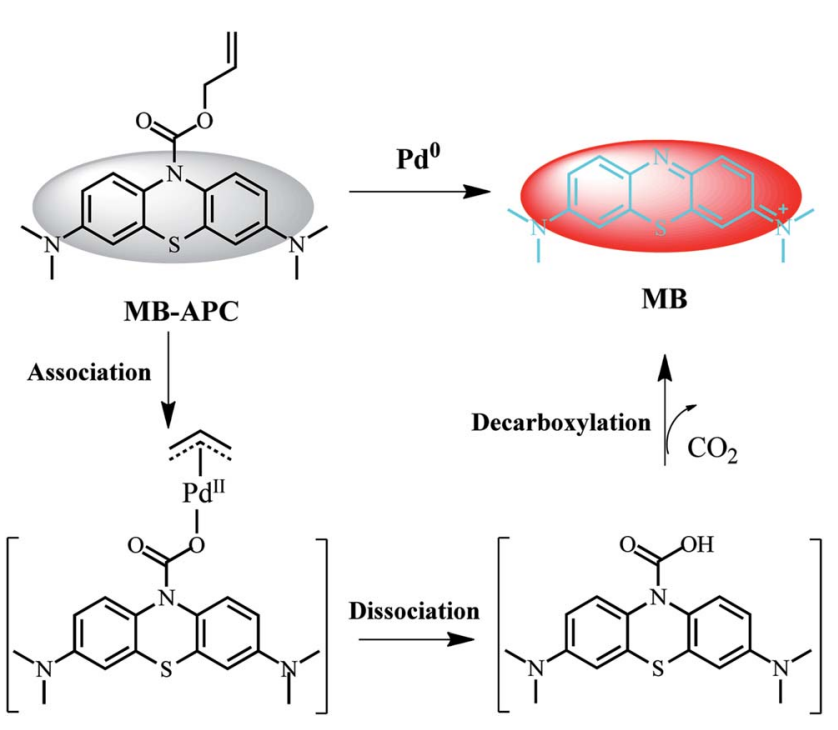

Scheme 1 Chemical structure and proposed sensing process of MBAPC for $\mathrm{Pd}^{0}$ based on Tsuji-Trost reaction. 
the image-guided surgery. ${ }^{19-22}$ However, the development of new fluorescent probes based on this NIR fluorophore has rarely been reported. ${ }^{23}$ It is well-known that MB could be converted to a colorless and non-emissive form called leucomethylene blue under reducing or acidic condition. To obtain the NIR fluorescent probe for $\mathrm{Pd}^{0}$, the allyl chloroformate moiety was introduced into the leucomethylene blue through a carbamate bond, leading to a stable colorless and non-emissive probe MB-APC. We speculated that MB-APC would be uncaged through the Tsuji-Trost reaction in the presence of $\mathrm{Pd}^{0},{ }^{24}$ leading to the releasing of free $\mathrm{MB}$, accompanied with color change and turnon NIR emission (Scheme 1).

\section{Results and discussion}

The time-dependent absorption and fluorescence intensity changes of probe MB-APC after adding 3 equivalent of $\mathrm{Pd}\left(\mathrm{PPh}_{3}\right)_{4}$ were evaluated to investigate its spectral properties and response to $\mathrm{Pd}^{0}$. As shown in Fig. 1A, MB-APC exhibited almost no absorbance in the visible region in the EtOH-PBS ( $9: 1, \mathrm{v} / \mathrm{v})$ solution. An absorption peak appeared at around $657 \mathrm{~nm}$ after adding 3 equivalent of $\mathrm{Pd}\left(\mathrm{PPh}_{3}\right)_{4}$ solution, which increased gradually with incubation time and reached the maximum values after incubation for $30 \mathrm{~min}$ at room temperature. In the fluorescence spectra, no emission could be observed for MB-APC solution (Fig. 1B), which was consistent
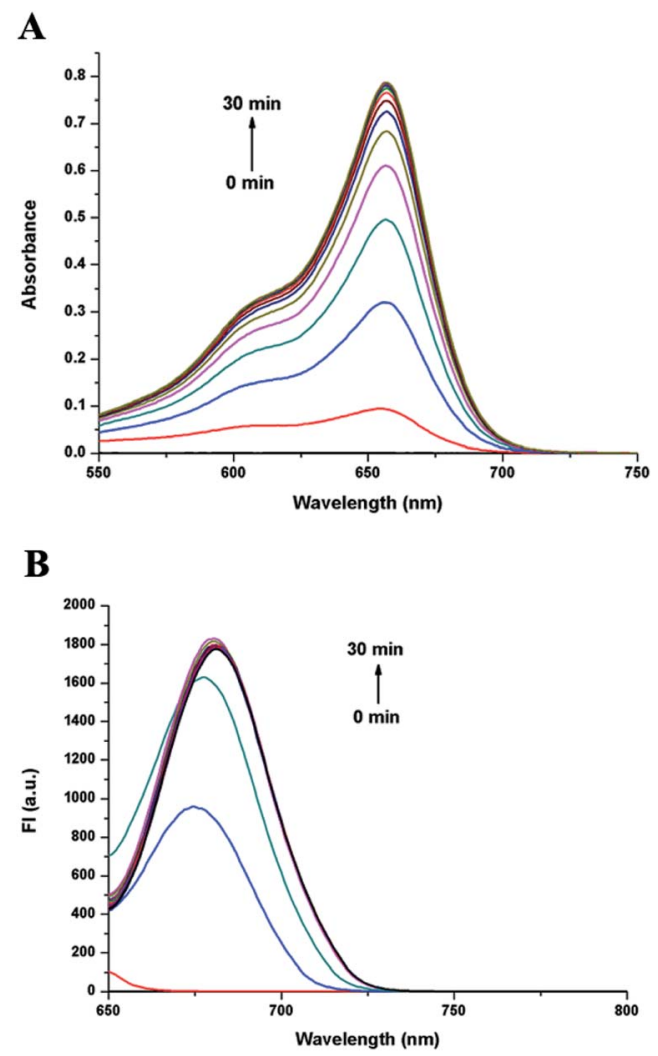

Fig. 1 UV-vis (A) and fluorescence spectral changes (B) of MB-APC (10 $\mu \mathrm{M})$ against time in the presence of $\mathrm{Pd}\left(\mathrm{PPh}_{3}\right)_{4}$ (3 equiv.) in $\mathrm{EtOH}-\mathrm{PBS}$ $(9: 1, v / v)$ at room temperature. with leucomethylene blue. After adding 3 equivalent of $\mathrm{Pd}\left(\mathrm{PPh}_{3}\right)_{4}$ solution, an emission peak at approximately $676 \mathrm{~nm}$ appeared and was significantly enhanced with incubation time, accompanied with slight red-shift. The emission peak was shifted to $681 \mathrm{~nm}$ and the fluorescence intensity reached a plateau in $30 \mathrm{~min}$ at room temperature (Fig. 1B). The turn-on fluorescence may be attributed to the releasing of free $\mathrm{MB}$ moiety through the broken of the carbamate bond, which was confirmed by almost the same absorbance and emissive peak with MB from commercial source (Fig. S4 $\dagger$ ), and the HRMS result (Fig. S5 $\dagger$ ). It is also noteworthy that both the excitation and emission of MB-APC reach the NIR range, which is very attractive for intracellular sensing and imaging.

To further explore the sensing ability of MB-APC to $\mathrm{Pd}^{0}$, UVvis and fluorescence titration experiments were carried out. As shown in Fig. 2A, the absorption band at $657 \mathrm{~nm}$ enhanced with the increasing $\mathrm{Pd}^{0}$ concentrations concomitantly. The significant absorbance enhancement (about 393 fold increase) was accompanied by a marked and vivid color change from achromatic to blue in ambient light (inset of Fig. 2A), demonstrating that MB-APC could be utilized as an on-site and naked-eye indicator for $\mathrm{Pd}^{0}$. Meanwhile, the linear equation was obtained. As shown in Fig. S6A, $\uparrow$ the plots of absorption fitted

\section{$\mathbf{A}$}

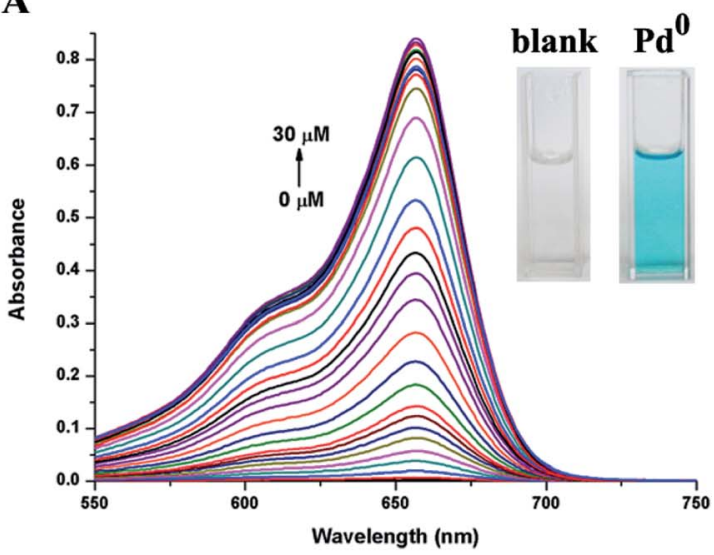

B

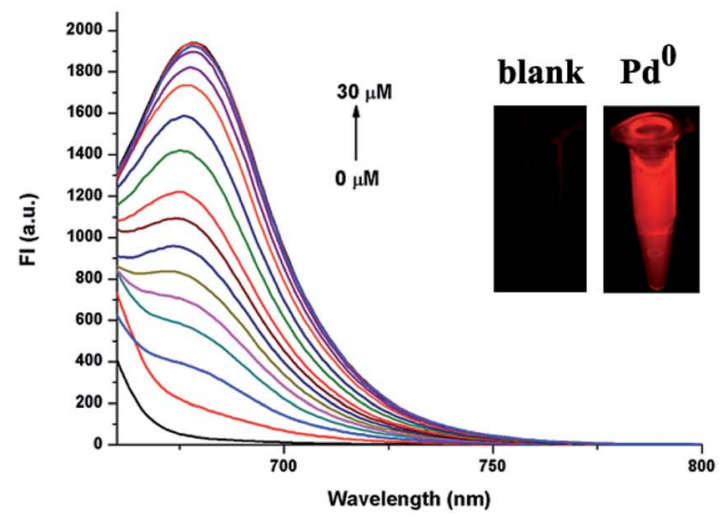

Fig. 2 UV-vis (A) and fluorescent (B) spectroscopic titration of MB$\mathrm{APC}$ by stepwise addition of $\mathrm{Pd}\left(\mathrm{PPh}_{3}\right)_{4}$. Conditions: $[\mathrm{MB}-\mathrm{APC}]=10 \mu \mathrm{M}$; $V_{\mathrm{EtOH}}: V_{\mathrm{PBS}}=9: 1,10 \mathrm{mM}$ PBS buffer, PH 7.4, $\lambda_{\mathrm{ex}}=650 \mathrm{~nm}$. The spectra were recorded at 30 min intervals. Inset: color and emission changes of MB-APC upon addition of $\mathrm{Pd}\left(\mathrm{PPh}_{3}\right)_{4}$. 
linearly with $\mathrm{Pd}^{0}$ concentration range of $0-6 \mu \mathrm{M}$ with a correlation coefficient of 0.994 . The detection limit of absorption for $\mathrm{Pd}^{0}$ was as low as $12.48 \mathrm{nM}$. Moreover, the enhancement (about 55 fold increase) of fluorescence intensity can be easily found upon addition of increasing amounts of $\mathrm{Pd}^{0}$, which could also be observed by the naked eye under the UV irradiation (Fig. 2B). As shown in Fig. S6B, $\uparrow$ the plots of fluorescence fit linearly with $\mathrm{Pd}^{0}$ concentration range of $0-2 \mu \mathrm{M}$ with a correlation coefficient of 0.994. The detection limit of fluorescence for $\mathrm{Pd}^{0}$ was deduced to be as low as $5.7 \mathrm{nM}$. The result indicated that MB-APC had low detection limit in both absorption and fluorescence, which illustrated that the probe can be utilized as an efficient tool for sensing traces of palladium in both colorimetry and fluorescence signal with high sensitivity. Compared with other reported fluorescent probes for $\operatorname{Pd}^{0}$ (Table $\mathrm{S} 1 \dagger$ ), NIR excitation and emission, and high sensitivity of MB-APC makes it a robust tool for sensing in both environment and biology samples.

To clarify the specificity of MB-APC towards $\mathrm{Pd}^{0}$ over other metal ions, the absorption and the fluorescent spectra response were further investigated upon addition of various metal ions to EtOH-PBS solution of MB-APC, respectively. 3 equivalent of $\mathbf{P d}^{0}$ and 10 equivalent of other metal ions were added to the detecting system. As expected, only $\mathrm{Pd}^{0}$ caused such remarkable signal changes in the both absorption and fluorescence spectra of MB-APC (Fig. 3). In contrast, other noble metal with catalysis such as $\mathrm{Ru}^{0}$, and other metal ions such as $\mathrm{Ag}^{+}, \mathrm{Fe}^{3+}, \mathrm{Hg}^{2+}, \mathrm{K}^{+}$, $\mathrm{Na}^{+}, \mathrm{Pb}^{2+}, \mathrm{Ca}^{2+}, \mathrm{Mg}^{2+}, \mathrm{Cu}^{2+}, \mathrm{Ni}^{2+}, \mathrm{Co}^{2+}, \mathrm{Ba}^{2+}, \mathrm{Zn}^{2+}, \mathrm{Mn}^{2+}, \mathrm{Pt}^{2+}$, $\mathrm{Cd}^{2+}, \mathrm{Al}^{3+}, \mathrm{Cr}^{3+}$, showed almost negligible disturbance to the spectra and color (Fig. 3), even up to 10 equivalent concentrations, indicating that MB-APC showed a notable selectivity to $\mathrm{Pd}^{0}$. MB-APC could efficiently discriminate $\mathrm{Pd}^{0}$ from other metal ions by the visual readout (Fig. S7 $\dagger$ ). Moreover, metal chelators such as EDTA have little influence on this detecting system (Fig. S8†). All these experimental results suggested that
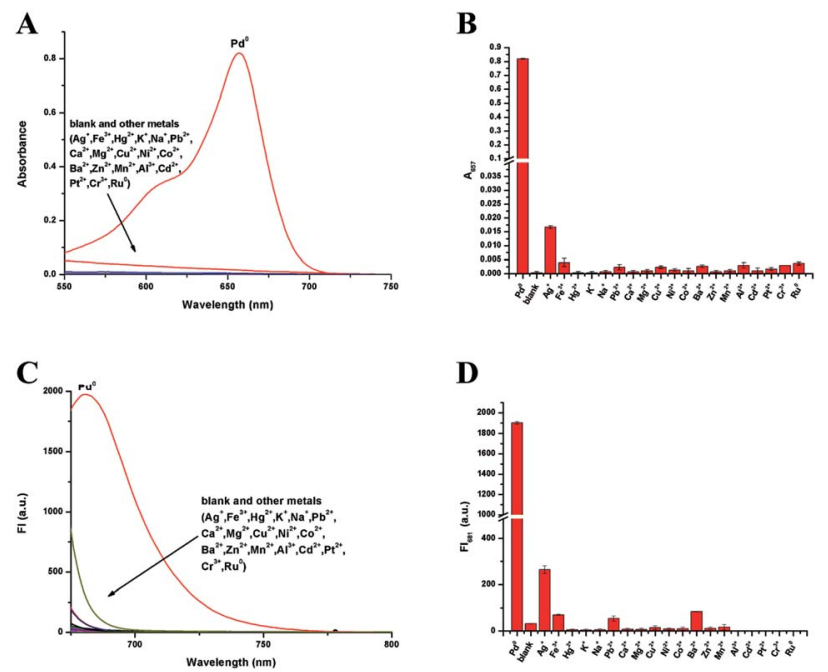

Fig. 3 UV-vis (A) and fluorescent spectra (C), absorbance at $657 \mathrm{~nm}$ (B) and fluorescence intensity at $681 \mathrm{~nm}(\mathrm{D})$ of MB-APC $(10 \mu \mathrm{M})$ in the presence of $\mathrm{Pd}\left(\mathrm{PPh}_{3}\right)_{4}$ (3 equiv.), $\mathrm{Ru}_{3}(\mathrm{CO})_{12}$ (3 equiv.) and the excess of representative metal ions (10 equiv.) in $\mathrm{EtOH}-\mathrm{PBS}(9: 1, \mathrm{v} / \mathrm{v})$ at room temperature. Each spectrum was acquired after mixing for 30 min.

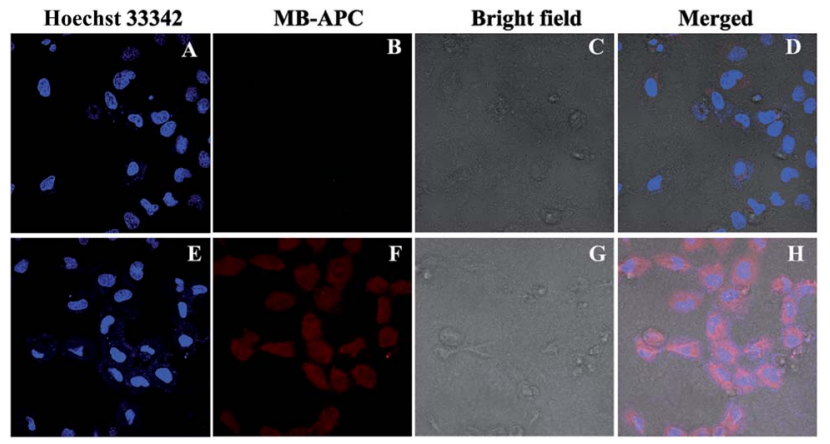

Fig. 4 Confocal fluorescence images of living HeLa cells using a $635 \mathrm{~nm}$ laser. HeLa cells were co-stained with Hoechst 33342 (10 $\mu M)$ and $M B-A P C(10 \mu M)$, without $(A-D)$ and with $(E-H)$ incubation of palladium $(30 \mu \mathrm{M})$ for $30 \mathrm{~min}$.

this probe MB-APC had an excellent selectivity for $\mathrm{Pd}^{0}$ over other metal ions.

Encouraged by the above findings, the potential intracellular applications of MB-APC were investigated using confocal laser scanning microscopy. We first studied the cytotoxicity of this probe toward using a standard MTT assay. The results revealed that exhibited very low cytotoxicity to living cells for $48 \mathrm{~h}$ even up to $100 \mu \mathrm{M}$ (Fig. S9†). Living cell imaging assay was conducted using HeLa cells. As shown in Fig. 4A-D, co-staining living cells using Hoechst 33342 revealed that free MB-APC exhibited almost no fluorescence signal, which was consistent with fluorescent spectra studies. By contrast, after incubation with $\mathrm{Pd}^{0}$ for $30 \mathrm{~min}$, a distinct strong emission response could be observed (Fig. 4E-H). The results demonstrated that MB-APC is both cell-permeable and capable of sensing palladium in living cells, which makes it a versatile tool for the detection of palladium species in environmental samples and in living cells.

\section{Conclusions}

In conclusion, a colorimetric and NIR fluorescent probe MBAPC for $\mathrm{Pd}^{0}$ was rationally developed. The distinct color change and turn-on NIR emission make it an on-site and visual indicator for $\mathbf{P d}^{0}$. MB-APC also exhibited high specificity and sensitivity, with the detection limit of $5.7 \mathrm{nM}$. In addition, MBAPC has been successfully applied in detecting and imaging of $\mathrm{Pd}^{0}$ in living cells, which revealed that MB-APC has the potential to track intracellular palladium species taking advantage of its specific turn-on NIR emission. All these results featured its promising application prospects for palladium sensing in both environment and biology field.

\section{Acknowledgements}

This work was financially supported by the National Natural Science Foundation of China (21502056), the Natural Science Foundation of Guangdong Province, China (2016A030310463), the Pandeng project for undergraduate in Guangdong Province (j2tw-k315001-16 to L. F. Z.) and National 
Undergraduate Innovative and Entrepreneurial Training Program (201610561163 to L. F. Z.).

\section{Notes and references}

1 R. R. Barefoot, Trends Anal. Chem., 1999, 18, 702-707.

2 K. Pyrznska, J. Environ. Monit., 2000, 2, 99N-103N.

3 K. Ravindra, L. Bencs and R. Van Grieken, Sci. Total Environ., 2004, 318, 1-43.

4 R. Merget and G. Rosner, Sci. Total Environ., 2001, 270, 165173.

5 J. Kielhorn, C. Melber, D. Keller and I. Mangelsdorf, Int. J. Hyg. Environ. Health, 2002, 205, 417-432.

6 B. Dimitrova, K. Benkhedda, E. Ivanova and F. Adams, J. Anal. At. Spectrom., 2004, 19, 1394-1396.

7 C. Locatelli, D. Melucci and G. Torsi, Anal. Bioanal. Chem., 2005, 382, 1567-1573.

8 K. Van Meel, A. Smekens, M. Behets, P. Kazandjian and R. Van Grieken, Anal. Chem., 2007, 79, 6383-6389.

9 H. Li, J. Fan and X. Peng, Chem. Soc. Rev., 2013, 42, 79437962.

10 M. P. Tracey, D. Pham and K. Koide, Chem. Soc. Rev., 2015, 44, 4769-4791.

11 J.-w. Yan, X.-l. Wang, Q.-f. Tan, P.-f. Yao, J.-h. Tan and L. Zhang, Analyst, 2016, 141, 2376-2379.

12 J. Zhang, L. Zhang, Y. Zhou, T. Ma and J. Niu, Microchim. Acta, 2013, 180, 211-217.

13 Q. Huang, Y. Zhou, Q. Zhang, E. Wang, Y. Min, H. Qiao, J. Zhang and T. Ma, Sens. Actuators, B, 2015, 208, 22-29.

14 L. Yuan, W. Lin, K. Zheng, L. He and W. Huang, Chem. Soc. Rev., 2013, 42, 622-661.
15 Z. Guo, S. Park, J. Yoon and I. Shin, Chem. Soc. Rev., 2014, 43, 16-29.

16 N. Ormeci, B. Savas, S. Coban, M. Palabıyıkoğlu, A. Ensari, I. Kuzu and N. Kursun, Surg. Endosc., 2008, 22, 693-700.

17 J. P. Tardivo, A. Del Giglio, C. S. de Oliveira, D. S. Gabrielli, H. C. Junqueira, D. B. Tada, D. Severino, R. de Fátima Turchiello and M. S. Baptista, Photodiagn. Photodyn. Ther., 2005, 2, 175-191.

18 M. Wagner, E. R. Suarez, T. R. Theodoro, C. D. A. S. Machado Filho, M. F. M. Gama, J. P. Tardivo, F. M. Paschoal and M. A. S. Pinhal, Clin. Exp. Dermatol., 2012, 37, 527-533.

19 Y. Ashitate, B. T. Lee, R. G. Laurence, E. Lunsford, M. Hutteman, R. Oketokoun, H. S. Choi and J. V. Frangioni, Ann. Plast. Surg., 2013, 70, 360-365.

20 A. Matsui, E. Tanaka, H. S. Choi, V. Kianzad, S. Gioux, S. J. Lomnes and J. V. Frangioni, Surgery, 2010, 148, 78-86.

21 F. P. R. Verbeek, J. R. van der Vorst, B. E. Schaafsma, R.-J. Swijnenburg, K. N. Gaarenstroom, H. W. Elzevier, C. J. H. van de Velde, J. V. Frangioni and A. L. Vahrmeijer, J. Urology, 2013, 190, 574-579.

22 E. Tanaka, F. Y. Chen, R. Flaumenhaft, G. J. Graham, R. G. Laurence and J. V. Frangioni, J. Thorac. Cardiovasc. Surg., 2009, 138, 133-140.

23 J. Bae, L. E. McNamara, M. A. Nael, F. Mahdi, R. J. Doerksen, G. L. Bidwell, N. I. Hammer and S. Jo, Chem. Commun., 2015, 51, 12787-12790.

24 F. Song, A. L. Garner and K. Koide, J. Am. Chem. Soc., 2007, 129, 12354-12355. 